\title{
Telemedicine in Gastroenterohepatology
}

\author{
Muharem Zildzic ${ }^{1}$, Nizama Salihefendic ${ }^{2}$, Ferid Krupic ${ }^{3}$, Emina Beganovic ${ }^{4}$, Lejla Zunic ${ }^{1}$, Izet Masic ${ }^{4}$ \\ Faculty for Health Sciences, University of Zenica, Bosnia and Herzegovina ${ }^{1}$ \\ Faculty of Medicine, University of Tuzla, Bosnia and Herzegovina ${ }^{2}$ \\ Department of Orthopaedics, Institute of Clinical Sciences, Sahlgrenska Academy, University of Gothenburg, Sweden ${ }^{3}$ \\ Faculty of medicine, University of Sarajevo, Bosnia and Herzegovina ${ }^{4}$
}

Corresponding author: Prof Muharem Zildzic, MD, PhD. Faculty for Health Sciences, University of Zenica, Bosnia and Herzegovina. E-mail: zildzic@yahoo.com

\begin{abstract}
Telemedicine itself is not the medical profession, it is not a medical specialty, but the way in which the medical profession conduct its activity. Therefore we are talking about tele otorhinolaryngology, tele cardiology or tele pathology. In the definition of a multitude of telemedicine that can be found in the literature is the following: Telemedicine is a system that supports the process of health care by providing ways and means for more efficient exchange of information that allows multitude of activities related to health care, including health care and health personnel, including education, administration and treatment. Telemedicine applications include: tele diagnosis, tele consultation, tele monitoring, tele-care, tele consultations and remote access to information contained in one or more databases. It turned out that telemedicine is an important factor in technological, professional, financial and organizational uniformity of development of the health system. Telemedicine, although a new area, to a large extent already changed the ways of providing health care, and even more influence on the ways of designing the future of medicine.

Key words: telemedicine, diagnostic discipline.
\end{abstract}

\section{INTRODUCTION}

Telemedicine is present in clinical medicine for the last 30 years, but has only begun to be significantly developed over the last five years. In its early stages of development, during the 60 s of the last century, the National Agency for Aeronautics and Space Administration NASA has played a major role in the development of telemedicine (1-10). Then the astronauts sent physiological reports to the Agency on the ground. That same year, NASA and the U.S. Health Service, began to provide health care to people living on Indian reservations in Arizona, using mobile rooms with ECG and x-rays, which were linked by satellite.

In 1967 is established a microwave link between the airport in Boston and Massachusetts General Hospital. During 70s was mentioned the possibility of using a color screen, or tele-dermatology for the treatment of skin infections during space flights.

At that time, the School of Medicine in Miami has enabled telemedicine for prisoners. In 1986, Mayo Clinic began to conduct two-way satellite program between Mayo camp in Rochester, Minnesota and Arizona, in order to assist physicians in remote locations. Although it can be said that telemedicine began with the invention of phone or wireless telegraphy, serious development of telemedicine, occurred, as has already been pointed out in the early 60s of the last century, when NASA as part of its project of space exploration Apollo began sending human crews in the space. Then began the development of telemedicine for the purpose of monitoring biomedical status and health of astronauts.

After NASA Space Shuttle project continued with the development and improvement of telemedicine applications during the cooperation of American astronauts and the United States with Russia at the Russian station Mir. At the same time NASA has continued to apply its experience in telemedicine in different areas of the world affected by natural and other disasters. In addition, was established telecommunications infrastructure, which should support further cooperation in areas including preventive medicine, public health, medical education, space biology, biological sciences, micro gravity science, space and aviation medicine and medical informatics. Initially set up satellite connection (768 kbps) to communicate through the Internet, with a plan to take over a hundred Russian universities connect with U.S. centers and engage in tele-education.

Another important factor in the development of telemedicine is the army. Historically, the development of international telemedicine was greatly influenced by the U.S. Department of Defense. As an example we can take the project "Akamai", a project of military medical center for electronic diagnostics and consultation at the air base, "Hickam" in Hawaii, has as its long-term goal to implement a telecommunication system for medical information, including PACS (Picture Archiving and Communication System), telemedicine, tele radiology, digital patient record and new tele surgery and tele pathological technology as soon as it becomes possible. Support system diagnostics based medical imaging has developed a Loral-Siemens. This system is a tele radiology system of radiology without film and transmission of digital images via satellite (U.S.) military hospitals around the world. 
Governments in developed countries today face major problems related to rising health care costs and demographic changes that have radically changed the health market. The health care systems around the world are experiencing a radical re-evaluation and reform. Objectives are usually multiple. For example, in the United States is pursued the realization of three objectives:

- Ensure universal access to health protection.

- Limit expenses growth.

- Maintain the quality.

The introduction of telecommunications technology enables sending of patient's data (instead of sending patients) at large, virtually unlimited distance. Send data to the experts who can help in solving health problems of patients in far shorter time than when instead of transmitting data the patient is transferred.

Escalating increase in health care costs attributed mainly to technological progress. Since telemedicine is expected to reduce these costs. One American study led by Arthur D. Little has shown that the introduction of electronic medical records, electronic referrals, electronic findings and video conferences can annually save more than 36 billion dollars.

The majority of active telemedicine projects are concentrated in the United States. As it is estimated that in the U.S. the costs of health care are growing about $25 \%$ in ten years (data for the period 1980 to 1990, Coles 1995), it is expected that the existing telemedicine projects before they are integrated.

Telemedicine projects are most numerous in the United States - where the federal government annually invests more than $\$ 100$ million in such projects.

Europe, namely the EU invests about 30 times less than the U.S. federal government, but the investment of various agencies and private investment within the EU are 3-4 times higher than the investment of the federal government of the United States.

Telemedicine projects covering different areas of medicine. Covering clinical, preventive, organizational or educational applications.

Regarding the state of telemedicine in some parts of the world, it can be said that the countries which comprise a large territory, are sparsely populated, are heavily involved in this business.

Australia is an example of a country which operates strongest on medical radio-communications network. Satellite links are used within the telemedicine project for the evacuation of patients (Royal Flying Doctor Service). Tele radiology, remote education and tele consultations have already become the practice in that country. The same can be said for the Nordic countries. Canada has established a teleconferencing system among universities since 1979. France in the 1984 at Telemedicine Institute (Toulouse) France established a computer network for the monitoring of infectious diseases, as well as being a pioneer in the development of health card (smart card) containing demographic and medical information about the patient, and read by a special reader that is connected to the computer. Japan is the leading country in the world when it comes to the use and de- velopment of PACS technology. Kyoto University developed the first telemedicine system to transmit images via television (HDTV), which uses ISDN standard for receiving high-resolution images.

In parallel with the increase in the number of studies on the possible use of telemedicine in the world, began the introduction of telemedicine in Croatia. At first it was just an area of scientific interest, but soon began its practical application.

The Ministry of Health established a tele radiology system in which, by means of a telecommunication system, in a unique network of 33 connected devices for computer tomography, digital subtraction angiography and magnetic resonance imaging in a total of 27 locations in 18 regional and district hospitals with a reference center in the Department of neurosurgery University Hospital Centre Zagreb (2, 3, 5, 6, 7). The system also includes 10 neurosurgical institutions that operate in seven medical centers (Zagreb, Osijek, Slavonski Brod, Rijeka, Pula, Split and Zadar) which allow the rapid exchange of information among neurosurgeons, and appropriate distribution of patients according to the degree of urgency and opportunities can be treated in closest neurosurgery institution.

The system is founded on the principles of enabling open access to the additional number of diagnostic units and functions, availability of diagnostic criteria necessary for the interpretation of the image that system conveys interactive communication and image manipulation, integrated archiving images and patient data, guaranteed confidentiality of stored data, and security training for all who use the system.

One example is the use telemedicine in internal medicine and is a project of the Croatian Academic and Research Network Teleconferencing which demonstrated the possibilities of application of telemedicine in Cardiac Pacing. Modern pacemaker has a complex diagnostic and telemetry functions that are realized by means of an external timer. Memory pacemaker allows arrhythmia monitoring and adjusting as a pacemaker also evaluation of drug therapy. In our patients every six months traveling in the center of electro stimulation for several hours to control carried out for about twenty minutes. Some failures of the system for stimulation and stimulation disorders occur so rapidly that they cannot be detected by regular inspection.

For complete safety of patients is necessary, therefore, to develop a reliable method of control at a distance. Teleconferencing has developed a prototype device in a remote outpatient programmable pacemaker and transmits ECG via Teleconferencing Network. In the center of electro workstation (client) associated with the device (server) via Teleconferencing Network, and on which the ECG monitor patients in real time and graphical interface controller of the pacemaker. Expert electro stimulation can from the center to the client to perform complete control patients at a great distance. Standard controller is connected by means of a specially developed interface with Sun Sparc workstation.

The graphical interface controller and display of ECG 
in real time have been developed for the remote workstation (client) using the Java programming language. The project has demonstrated that the transmission of the ECG in real time is possible from Split to Zagreb and has successfully completed reprogramming of the pacemaker in Split from Zagreb $(2,3)$.

Telepathology for a number of reasons represents one of the most competitive segments of telemedicine. In Croatia, tele pathology began to develop in 1993 in the direction of the tele pathology system and in the direction of software development.

Given the circumstances to set the system works with still images and the possibility of interactive communication using ordinary telephone lines or the Internet. The development of national communication channels of higher speed (ISDN and ATM) will enable the acceleration of the whole process $(2,3)$.

The application of tele-pathology in Bosnia and Herzegovina $(\mathrm{B} \& \mathrm{H})$ began in late 1996 the Institute of Pathological Anatomy, Faculty of Medicine in Sarajevo, in cooperation with the Department of Pathology $(1,10$, 11), Faculty of Medicine in Zagreb. In this project, apart from these two institutions involved in the project was the Institute of Radiology and Oncology and Department of Ophthalmology, Clinical Center University of Sarajevo. The first steps in introducing tele-neurosurgery in Bosnia and Herzegovina started in 2005. During same year was installed Vamstec station in order to provide communication between the Department of Neurosurgery in Sarajevo and Cantonal Hospital in Travnik. Telemedicine in Bosnia and Herzegovina exist and there are more and more users, but at this time the global application is not possible without a wider involvement of all stakeholders in the health system, as well as the adequate financial support (4).

Today, many countries are faced daily with increasing health care costs and demographic changes, especially the aging of the population, leading to radical changes in health care markets. On the other hand, the spatial distribution of the population varies from country to country and even within one country.

Population of an area depends primarily on the natural environment. Greater isolation areas entail a lower population density.

Isolated areas are islands, mountain regions, regions where the human hand has not done what attracted population, on the climatic conditions, or something else.

The health care systems around the world are experiencing a radical re-evaluation and reform, especially when it comes to health systems in countries in transition.

The objectives of such re-evaluation and reform are manifold, although they may be reduced to three key ones:

- Ensure the availability of health care.

- Limit costs increase.

- Improve the quality of or at least maintain it.

The introduction of telecommunications technology to send data on the patient instead of the patient, to the large, virtually unlimited distances. Under virtually unlimited distances are thought to date back to the distance to which the capabilities of modern telecommunications technology, which means that the patient can be even in space.

Via telecommunication links data can still be sent to experts who can help solve the health problems of patients in far less time than would be possible if the data transmitted rather than patients. In addition, the transfer or transport patients can sometimes be risky.

Escalating increase in health care costs attributed mainly to technological progress. When all the technological advances wanted to approach anyone, even those in sparsely populated areas, would require a lot of resources (equipment and personnel) that would be difficult to any country, no matter how rich and developed, could provide. Since telemedicine is expected to reduce health care costs. One American study led by Arthur D. Little has shown amazing result: the introduction of electronic medical records, electronic referrals, electronic findings and video conferences can annually save more than 36 billion dollars. Navein and colleagues in their study showed that in Europe many conventional consultations could replace tele consultations. Potential savings would amount to 3.7 million dollars for travel expenses and savings 25,000 working days-for an estimated 31,000 consultations a year.

Pilot study by Tuulonen and colleagues from Finland has shown that using tele ophthalmology in the case of glaucoma, only in travel expenses, could save about $\$ 55$ per visit.

Speed in the provision of health services and the availability of experts at home and abroad is expected to result in better service and higher quality.

Telemedicine however should not only be used as curative. Preventive telemedicine also can and should be represented in telemedicine activities. The saying "prevention is better than cure", "copied" in telemedicine activities, born tele preventive medicine. Tele preventive medicine focused towards population-education and public health measures, and efficiency can be achieved with a smaller investment than is the case with clinical telemedicine.

Education is a key element in the telemedicine infrastructure. It is not enough just to be able to use telemedicine application, it is necessary to understand the capabilities of information technology and modern information systems. It is necessary to identify the specific educational groups, for example, that the doctors/ dentists, nurses/technicians, managers in health care administrators in the health, medical informatics and general IT-specialists in the field of computer science (as recommended by the International Association for Medical Informatics - IMIA International Medical Informatics Association), and establish appropriate educational programs for each group separately.

\subsection{Public health telemedicine}

Global Health Network-GHNet conceived in early eighties as part of a project about diabetes in children by World Health Organization, is actually a product of 
the initiative of the Institute of Epidemiology, School of Public Health, University of Pittsburgh. The main setting for the construction of a global health network was as follows:

Establish a permanent international health telecommunications networks in order to improve direct communication between the medical staff in the region and beyond, to encourage the development of national telemedicine and telematics networks. Network should develop and improve medical and public health collaboration by braking down language, cultural and political/ bureaucratic barriers.

Such permanent international medical telecommunications network should provide (a) the exchange of public health information; (b) public health and other health education activities; (c) the prevention and/or mitigation of diseases and disasters, (d) planning response to outbreaks and disasters, and (e) responses/ actions on the occurrence of disease and disaster.

National and international medical and public health actions should be improved with a focus on training at the national level, particularly training in rapid assessment of the health situation after the disaster, and use of portable stations in the areas of high risk which would allow connection via satellite.

To realize the medical and public health collaboration regardless of language, cultural, political and bureaucratic barriers, and permanent international medical telecommunications networks must be developed in collaboration with WHO, Pan-American Health Organization and other inter-governmental and international non-governmental agencies with global and regional medical and other interests and responsibilities. These agencies must encourage the development of multilateral, regional and the international agreement to common action.

International telecommunications network must be technologically gradually develop. It should identify something, such as clearing center for information about new technologies and their application to global standards in telemedicine.

The result of the implementation of the network based on these settings is the website (http://www.pitt.edu/ HOME/GHNet/GHNet.html) where you can find many educational materials that provide continuing education of public health workers, students and the laity. $(5,6,7)$

\section{TELEMEDICINE IN}

\section{GASTROENTEROHEPATOLOGY}

Gastroenterology is the branch of internal medicine. Gastroenterology studying diseases of the digestive system and diseases of the esophagus, stomach, small intestine, colon, biliary tract, pancreas and peritoneum $(9,10)$.

\subsection{Tele education in gastroenterology}

Internet and the computerization of the entire society have dramatically changed the daily work and communication between people. It has developed into the most powerful knowledge base, with an unusually large amount of information available to each individual. Suffice it to mention that according to some estimates, a day on the Internet create 7 million new pages (this includes only static Web pages that are accessible to common search tools, while the overall number of new pages is even several times higher. Such expansion of the source and availability of data also influenced the development of telemedicine.

In the U.S., approximately 60 million people find "online" information about the health since the 1998, more than a quarter of gastroenterological patients searched the information about their illnesses on the web, and it is calculated that more than two-thirds in the future will use the web as a source of information. In fact, after learning from a computer, which is a novelty of the 20th century, at the beginning of the 21st century, the emphasis in education is placed on learning through the Internet. It occupies a special place in continuous medical tele education, "on-line" education or "distance learning”. Specifically, continuing medical education, and its evaluation process of collecting points required for relicensing the duty of physicians who work in direct health care jobs in the world. With the help of the Internet or the Web-the most popular web service for search and display information-can bypass the limitations of previous models of continuing medical education. The traditional forms of continuing medical education, such as organizing training courses, seminars, symposia and conferences, limited by time and place of maintenance, space, performance and price (arrival of participants on the course from distant places, accommodation, absence from the workplace, financial conditions and so on). Therefore, to exploit the potential of the Internet for educational purposes is a new challenge for teachers of medicine. Today, at the Internet can find numerous websites that contain courses of continuing medical tele education in the world (e.g., eMedicine, CMEcourses or it is possible to follow the professional news via specialized web portals like GastroHep or Medscape/Gastroenterology).

With the World Wide Web, also other network services can improve and facilitate communication and education among physicians. This particularly applies to email and web forums.

Telemedicine with tele education is additionally important in diseases with high public health importance, such as diseases of the gastrointestinal, hepatobiliary and pancreatic systems.

Using the web within tele education in gastroenterology is possible in several ways. The first way is a copy of the classic medical education: view teaching materials, assessment "online" and if the user is successfully solved test, gets a certificate and the number of points according to the practice of the competent Chamber of Physicians. Another way is to visit specialized websites (Gastrohep, Gastrosource) that offer continuing medical education related to problem cases from practice. However, today's technology in gastroenterology, like video endoscopy, allows storing images in digital format on a computer and its replay. Together with other 
clinical and laboratory relevant data, such materials can be stored on a computer connected to the Internet (Web server) and used for continuing medical education of physicians gastroenterologists. Such integration of audio-visual clinical data and their archiving the web servers make meaningful basis for electronic education in gastroenterology (8).

\subsection{PACS (Picture Archiving and Communication System)}

Many if not most hospitals (in developed countries) applied PACS (picture archiving and communication systems) technology $(9,10)$. PACS is a modern, computer alternative to paper and filmed archive. It is an integrated system consisting of devices for medical diagnostics, servers, workstations for data access, computer networks that connects the system components, databases and interfaces to other systems (e.g., hospital (inpatient) and radiology information systems - HIS and RIS). They base this system on web technology allows data to be accessed from different locations within the medical facilities, as well as from remote locations outside of the institution. That system unites the functions tele-radiology service and a system for archiving, searching and viewing of medical images and patient data.

PACS stores images from different medical devices (modalities): ultrasound, magnetic resonance (MR), PET scanners, CT scanners, endoscopy (ENDO), mammograms (MG), digital radiography (DR), computed radiography (CR), nuclear medicine (NM).

PACS architecture consists of a server (a central computer) in which there is PACS software for managing medical examinations, and the client (CT scanners, PET scanners, ultrasound devices and computers). The recordings are stored in the DICOM format (standard). On client computers is DICOM viewer software that allows you to view medical images.

Recordings from CT scanners, PET scanners, and ultrasound equipment, with the help of the DICOM standard, are sent to the server using the DICOM C-Store protocol, and then the server archive footage. Server and client communicate using the DICOM protocol. Client computers with the help of special software "DICOM Viewer" allows review of medical images, and there is also the possibility of commenting findings.

Every computer in the PACS network is identified by its network address, the communication port (TCP/IP), and named. In addition to working with PACS is necessary to provide a larger capacity hard drives, as well as modern monitors, to display the images were of better quality. Monitors with 3 megapixels, are required to read standard x-ray images, and monitors with 5 megapixels are needed for review mammograms.

There are six basic elements of such a system:

- Image acquisition - Acquisition of digital images requires the existence of medical devices with corresponding interface to their PACS system.

- Communication network - As a basis PACS system exists communication network for transmission of images and their associated data. The structure of the network has a decisive influence on working speed of the whole system. The network function of PACS systems requires both LAN and WAN environments.

- Image display - workstation for viewing images are usually in doctor's office. They must meet certain quality. Quality of workstations is reflected in the physical characteristics of the monitor.

- Data on patients - Hospital IT system of the HIS (Hospital Information System) and radiological IT system or the RIS (Radiology Information System) must have interface to a PACS system. Standard that allows is HL7. HL7 (Health Level Seven, 7th OSI Layer Protocol) is a standard for the electronic exchange of information between the medical applications on the seventh layer of the OSI model.

- Image archive - The system for archiving recordings should be centralized, with support for DICOM and HL7 standards.

- Web server - An application that is located on the web server should provide access and adequate representation of data employed in the medical center and the remote users who need to enable access to all or only certain part of patient's data.

PACS is mostly used in radiology and PACS provided savings in this area are significant. Once the images were store under the radiological departments and it led to overcrowding and disorganization. Today, these recordings are kept electronically on a computer, and the savings in space is very important. Thanks to PACS, there is no more need for the preparation of expensive images, because the recordings stored on disks.

Acquisition devices and rarely communicate can make use of $10 \mathrm{Mb} / \mathrm{s}$ transmission. Workstation and server is running with $100 \mathrm{Mb} / \mathrm{s}$. For the increasing number of computers may Gigabit Ethernet $(1000 \mathrm{Mb} / \mathrm{s})$ to replace the $100 \mathrm{Mb} / \mathrm{s}$, but this too will improve work. What is needed is "smarter" architecture.

Closely carrying out medical consultations remotely and transfer static medical imaging. Using computer network it is possible that doctors come up with an online image storage, image sent specialists to interpret (a form of consultation on the remote), or to receive information from ambulances. In a central position in all this occupies PACS - Picture Archiving and Communications System.

The potential benefits of such systems may be the following: improved handling and use of medical imaging (i.e., reducing the possibility that the image is lost for incorrect or misguided). At UCSF have noticed that before the introduction of PACS-and between $15 \%$ and $20 \%$ radiographic images were lost, and that hundreds of other shots unviewed, improved quality of care through the interpretation of experts and reduction of costs radiological interpretation.

The main technological challenge of tele imaging is a size of static images. Typical uncompressed digital medical images have a size of about $25 \mathrm{kB}$ (nuclear medicine) to $50 \mathrm{MB}$ (digitized mammography), but it is often 
necessary to do repeated recording (from different angles or to permit comparison of images from the same perspective), so that the total size of uncompressed data from 1-2 MB to almost $200 \mathrm{MB}$.

Flow required for the transmission of these images depends on several factors, in particular, is essential: the period during which the transfer must be completed and allowed the level of compression (not allowing the degradation of the image so that call into question the interpretation and diagnosis). Compression techniques without losing reducing the image size 3-4 times and compression with loss of image can be reduced 10 to 20 times without loss of diagnostic image quality in some applications.

During applications is also essential protection of data, because during transmission and storage of images must be preserved the confidentiality of the patient and the integrity of the data. Confidentiality of personal data can be achieved using the techniques of user authentication, access control and data encryption. Data integrity can be preserved (an essential feature of a digital image is that it can be easily changed) digital signature technologies. It is necessary to find compromise between these demands, costs and ease of use.

\subsection{DICOM standard}

DICOM standard is a set of rules that allows the exchange of medical recordings and information between computers and hospitals. Standard establishes a common language, which allows capturing of medical information, which is made using one manufacturer equipment that can be used in digital systems from other manufacturers.

DICOM is not just a file format. DICOM enables data transmission, storage and is designed to cover all functional aspects of medicine in the field of digital images. Also, the PACS information systems are always mentioned when talking about the DICOM standard. They are directly related to the DICOM standard. PACS systems are medical information systems, which are designed to store, distribute and display digital medical images. Thus, they provide an overview of medical images from CT scanners, PET scanners, ultrasound equipment, etc. It is difficult to imagine modern medicine without DICOM and PACS.

All data in the real world: the patients, studies, medical equipment, DICOM seen as objects with the appropriate properties, or attributes. DICOM calls this IOD (Information Object Definition). Thus, the IOD is a collection of attributes that describe an object. IOD patient can be: name and surname, date of birth, weight, etc. In a broader sense (patient like any other DICOM object) consists of a set of attributes, however, maintains a list of all DICOM standard attributes (more than 2000), better known as DICOM vocabulary data in order to ensure their consistency in naming attributes.

When new recordings are made, they can be transferred between different DICOM devices and applications. DICOM this process of "requirements" and "sharing" of data calls a service-rendering model, in which the DICOM applications provide services to each other. DICOM these associations calls SOP (ServiceObject Pairs) and regroup them in the SOP classes. For example, keeping the CT images from a digital CT scanner for digital PACS archive, reports CT to SOP for storage. The CT scan is a DICOM IOD (DICOM object data).

CT scanner service calls for storing the archive, and then the archive provides a service for storing to a scanner. The difference between the service request and service that provide services, called DICOM Service Class Users (SCU) and Service Class Providers (SCP). In this example, the CT scanner is a user - SCU and a digital archive is a server-SCP.

Each network exchange between SCU and SCP is called association. Each network data transmission begins with the establishment of associations-DICOM conversation when two applications exchange data. Many DICOM devices, and applications, produced by different manufacturers, and therefore it are important that any DICOM device is accompanied by its DICOM report about the conformation of the manufacturer. This report explains that SOP services the device supports, and to what extent (if supported by the SCU or SCP or both services). So DICOM report on conformation presents basic instruction on any DICOM device. For example, if a hospital buys a digital archive that supports only service for storage (CT Storage SCU), and is not supported by the SCP service for storage (CT Storage SCP), the hospital will not be able to store CT images. The digital archive will not be able to provide CT services for storage $(9,10,11,12)$.

Conformance Statements are critical for interoperability, which is the main purpose of the DICOM standard. They provide important information for implementation and integration in order to establish interoperability between applications. In the event that interoperability is not established, may be of importance for finding potential causes.

Organization of the DICOM has defined by the minimum necessary requirements for suitability to standard and method of reports implementation as described in Conformance Statements. This document does not provide any information about testing and validation, as well as on the selection of optional features, class of service, or information objects for a specific device. The choice of components in DICOM standards that could be useful in the implementation depend on the application. DICOM information objects or formal definition of information objects (IODs) are used for the description of real objects. The patient is by definition a person who receives or is registered to receive health care services, or is the subject of one or more studies for any purpose, such as clinical research. If the patient is a real object that we want to describe the use of the DICOM standard, it is necessary to define the IOD patient or set of attributes (ID (identification) number, name, sex, height, weight, etc.) that represent the structure of DICOM patient adapted environment. These attributes are, still, confer specific value, which is determined by 
a specific real property. Each attribute has its own label (Tag), which uniquely identifies it.

Information objects describe real objects using sets of related attributes. These sets of related attributes are called modules and they describe a class of real objects. One of IOD's is the IOD SC (Secondary Capture) images, describing images that are converted from non DICOM into DICOM format, regardless of the modality. An example of such an image is an image obtained by film digitization systems that convert analog format to a digital or scanned document, which includes handwriting. IOD SC image can be described using five information entity (IE): the patient, test, course, equipment and files. Each information entity is described by appropriate modules, which are associated with specific attributes. Awarded attributes may be required, optional or conditional.

IOD, which is generally used to represent one class of real objects, is called a normalized IOD. Then IOD refers to a single entity in the DICOM model of the real world. In contrast normalized, IOD which includes information about related objects in the real worlds is called the Composite IOD. These IODs consist of attributes that can be separated within the IOD, but not in relation to the related real objects. This gives context information sharing.

In addition to the communication model, information model of DICOM standards is very important. For information object definition applies to a group of service (DSG), let's say DIMSE services. It can be said that the SOP Class, Class of pairs service-object described by IOD and service groups that are applied over the IOD infection. SOP Class IODs and may be composite and normalized. Normalized SOP Class is defined as the union of normalized IOD and a set of matching DIMSE service (DIMSE-N). Similarly, composite SOP Class is defined as the union of a composite IOD and a set of matching DIMSE Service (DIMSE-C).

DICOM uses different data elements and their coding. Using concrete presentation of types of values (VR) represent the elemental data. VR can be a date, time, integer, string and other. In practice, the result of the DICOM standard is an image in DICOM format. DICOM image is demanding for handling and archiving and is the most common example of DICOM files. The file format that meets defined standards (PS 3.10) is called the file in DICOM format or just a DICOM file and its extension is .dcm. DICOM image format consists of a header and the image data. The header can contain a lot of information that are relevant and accompanying image. High quality medical images are essential. It is also essential quality preservation headers in a format that is common to all applications [GUL02].

DICOM files usually cannot "directly" be viewed at display, but need software support in the form of a DICOM viewer. Media such as a CD or DVD with DICOM images typically contains: a program for automatically launching application, DICOM viewer for viewing files, folder with DICOM images and DICOM directory compiled by the index and all the relevant in- formation about DICOM images that are in the directory. When DICOM viewer is not available it is necessary to download additional software packages $(9,10)$.

\section{CONCLUSION}

It turned out that telemedicine is an important factor in technological, professional, financial and organizational uniformity of development of the health system. Telemedicine, although a new area, to a large extent already changed the ways of providing health care, and even more influence on the ways of designing the future of medicine. Regardless of whether it will, as it has been so far, the technological reality of multiple outperform optimistic expectations, it can be safely assumed that telemedicine in the coming period will be one of the areas that will most contribute to medical science and practice. The application of telemedicine in gastroenterology can increase the efficiency and productivity of health care and health insurance, we can monitor and evaluate the work, eliminate unnecessary duplication of collected data, and the data can be comprehensively used, as well as increase the quality and efficiency of health care of patients.

\section{CONFLICT OF INTEREST: NONE DECLARED.}

\section{REFERENCES}

1. Masic I. History of Informatics and Medica informatics. Avicena. Sarajevo, 2013.

2. Kovačić L, Telekomunikacije u zaštiti zdravlja; Telemedicina u Hrvatskoj. U: Richter Branimir (ur.) Akademija medicinskih znanosti Hrvatske, Zagreb, 1998.

3. Kern J. Zdravstvo digitalnog doba. HCJZ 2005; 1(3). Dostupno na: http://www.hcjz.hr/clanak. php? id $=12548$

4. Mašić I, Novo A. Telemedicine in Bosnia and Herzegovina. Acta Inform Med.. 2006; 14(2): 103-105.

5. Kern J, Kovačić L. Telemedicina: što - zašto - kako? U: Klapan I, Čikeš. Telemedicina u Hrvatskoj. Zagreb: Medika. 2001: 6-20.

6. Kern J, Kovačić L, Orešković S. Telemedicina i javno zdravstvo U: Kurjak A, Richter B. Telemedicina u Hrvatskoj. Dostignuća i daljnji razvitak. Zagreb. Akademija medicinskih znanosti Hrvatske; 2001: 247-259.

7. Pale P. Stanje i strategija razvitka infrastrukture za telemedicinu. U: Richter B, ur. Telemedicina u Hrvatskoj. Zagreb: Akademija medicinskih znanosti Hrvatske; 1998: 31-44.

8. Pulanić R, Iveković $H$, Pulanić D, Vražić $H$, Ostojić R, Pemužić M. i sur. Prva „on-line“ trajna edukacija iz gastroeneterologije u Hrvatskoj. Liječ Vjesn. 2003; 271-273.

9. Masic I. Telekonsultacije u Urgentnoj medicini. U: Salihefendic N, Zildzic M, Licanin Z, Muminhodzic K, Zerem E, Masic I. Akutna abdominalna bol. Avicena. Sarajevo, 2005: 145-170.

10. Masic I. Telematics, Telemedicine, Tele-education. U: Masic I, Ridjanovic Z, Pandza, H, Masic Z. Medical informatics. Avicena. Sarajevo. 2010: 351-384.

11. Trajković G. Telemedicina. Dostupno na: http://mfub. bg.ac.rs:

12. Reljin I,Gavrovska A. Telemedicina. Akademska misao, Beograd. 2013: 61-95. 